\title{
Alicja Krella
}

The Szewalski Institute of Fluid-Flow Machinery, Polish Academy of Sciences, Cavitation Group, ul. Fiszera 14, 80-231 Gdansk, Poland,

\section{THE EXPERIMENTAL RESISTANCE PARAMETER FOR TiN COATING TO CAVITATION ACTION}

\begin{abstract}
A series of cavitation erosion tests were carried out to investigate the resistance of the TiN coatings to cavitation action. The TiN coatings were deposited at various deposition parameters on austenitic stainless steel, X6CrNiTi18-10, and with various thickness by means of the cathodic arc evaporation method (ARC PVD). Investigations were performed in a cavitation tunnel with a slot cavitator. The investigations show that hardness, Young's modulus, adhesion and coating thickness have an influence on coating endurance to cavitation degradation. The obtained parameter $\mathrm{H} \cdot \mathrm{L}_{\mathrm{C} 2} / \mathrm{E} \cdot \mathrm{h}^{1 / 2}$ shows very good fitting to date points $\left(\mathrm{R}^{2}=0.96\right)$. With the increase of the $\mathrm{H} \cdot \mathrm{L}_{\mathrm{C}} / \mathrm{E} \cdot \mathrm{h}^{1 / 2}$ parameter the mass loss decreases indicating a continuous improvement in the cavitation erosion resistance.
\end{abstract}

Key words: nanocrystalline TiN coatings, cavitation erosion, degradation, fracture, impact

\section{INTRODUCTION}

Cavitation phenomena occurs in fast flowed liquid through a barricade which disturbs the flow and causes the rapid pressure degrease under the critic pressure. Moreover, all liquids contain some dissolved gas, which is the source of cavitation nuclei. If the liquid pressure exterior to a nuclei/bubble of radius $\mathrm{R}$ will be lower than the saturated vapour pressure, the nuclei will be activated and the radius of the bubble will increase. The number and magnitude of cavitation bubbles depend on the level of the decrease of the water pressure and nucleation sides in water. Brennen [1] showed that in the cavitation cloud are $10^{9}-10^{14}$ cavitation nuclei and the number of cavitation nuclei increases along with its radius decreases. The increase of the liquid pressure will result in the cavitation bubble collapse. Cavitation bubbles collapse violently emitting either the micro-jets or shock waves with high velocities, pressures and temperatures. Impacts of shock waves and micro-jets are the source of the noise and material damage. Knapp [2] assessed that only one of 30,000 cavitation bubbles is able to cause plastic deformation (a pit) of soft pure aluminium.

Philipp and Lauterbour [3] investigating the implosions of cavitation bubbles introduced parameter $\gamma=\mathrm{S} / \mathrm{R}$, where $\mathrm{s}$ is the distance from the centre of cavitation bubble to a solid surface and $\mathrm{R}$ is the maximum radius of cavitation bubble. They noticed that bubbles generated in the ranges $\gamma \leq 0.3$ and $\gamma=1.2$ to 1.4 were the most aggressive and caused the greatest damage, because the jet hit the solid boundary directly with maximum speed without being decelerated by a water layer. The cavitation bubble of radius $\mathrm{R}=1.45 \mathrm{~mm}$ impacted with a maximum speed of $200 \mathrm{~m} / \mathrm{s}$ onto the solid boundary. Calculations 
performed by Bourne [4] show that the impulse pressure might reach the pressure of $\mathrm{GPa}$ and velocity impact might be as high as $600 \mathrm{~m} / \mathrm{s}$. This group of impacts act in a very short time in the range from nanoseconds to tens of microseconds and with high dynamic force. When a dynamic force impacts on the surface of a material, it initiates stress in the impact zone. At the moment of impact the first plane of atoms on the material surface is accelerated inwards and cause elastic respond in material. The stress will propagate as a wave. Moreover, if stress in the impact zone exceeds $3 R e$, where $R e$ is yield stress, then locally plastic flow will begin, according to Tabor theory [5]. In addition, high loading rate causes high strain rate and, as a consequence, an increase of elastic-limit stress. This is connected with the speed of dislocation generation and motion. Just after few nanoseconds of shock loading the phase transformation and dislocation activity may occur [6]. The dislocation investigation shown in Ref. [7] revealed that in X6CrNiTi18-10 stainless steel after 5 minutes of exposition to cavitation attack arose the dense dislocation network, the dislocation cell structure, slip bands crossed by adiabatic shear bands and dislocation loops in the area where the highest cavitation pulses acted. What's more, if the stress wave reaches the phase interface, or the surface of new material, like in coated materials, part of the stress wave will further transmit into the second phase, while the rest will be reflected, forming a secondary wave. On the other hand, all discontinuity spots are the places of stresses and dislocation pile-ups. Thus, they are also the places of nucleation and development voids within the material. As a consequence, this leads to micro-cracks initiation. The processes such as fracture or adiabatic shear require times of around $10 \mu \mathrm{m}$. Due to all mention actions the stress wave decays and finally vanishes. Thus, the respond of the material is spatially limited and temporary.

When the distance of bubbles implosion was in the range between $\gamma=1-2$, then the velocity of the jet was still very high, but the water layer between bubble and solid surface decelerates it and the final impact velocity was smaller than the maximum velocity of the jet. The jet velocity at the moment of impact onto the solid surface is in the range from $120 \mathrm{~m} / \mathrm{s}$ to $20 \mathrm{~m} / \mathrm{s}$ [3]. The jet still impacts with high dynamic force and initiates stress in the impact zone. Stress in the impact zone might exceed $3 R e$, but also can be under Re. Thus, the elastic-plastic deformation can occur. Because the loading rate is still high and the loading time is much longer than in previous case, the dislocation generation and motion occurs causing an increase of dislocation density and voids formation in the zone of action. As a consequence of the increase of dislocation density, the increase of elastic-limit stress and of the strength of material occurs. In Ref. [7] was revealed that in X6CrNiTi18-10 stainless steel after 5 minutes of exposition the dislocation network arose, as well as, the slip bands, dislocation loops and the dislocation cell structure, but with cells larger than in previous case. Besides, all discontinuity spots are the places of stress and dislocation pile-ups. Thus, they are also the privileged places for void formation and micro-cracks initiation. Due to all mention processes the respond of the material is spatially limited and the stress wave finally vanishes.

When the distance of bubbles implosion was further than 2R $(\gamma>2.2)$, the influence of water layer increases and, according to Philipp and Lauterbour's measurements [3], the impact velocity of the jet will fall down below $20 \mathrm{~m} / \mathrm{s}$. Low dynamic force connected with long time of impact causes mainly elastic response of the material.

Thus, the place where the bubbles implode had significant influence on impact velocity and the duration of a loading impact, which is also depended on impact velocity. 
Cyclic impact of cavitation pulses onto a material surface causes its gradual degradation called "cavitation erosion". The degradation process consists of three main stages (Fig. 1): incubation (I), acceleration (II) and steady state (III). In the incipient stage of erosion, incubation period, the slip bands and plastic deformation of the material surface in the form of pits occur. The level of deformation increases with time. Repeated impacts of cavitation pulses lead also to hardening the surface layer and initiation some micro-cracks. Later on, micro-cracks develop into macro-cracks, they join and finally cause the spalling and consequently the mass loss. During acceleration period the race of creating new cracks increases as well as the moved spalls. The geometry of the material surface changes, what has an effect on degradation process and leads to steady state period.

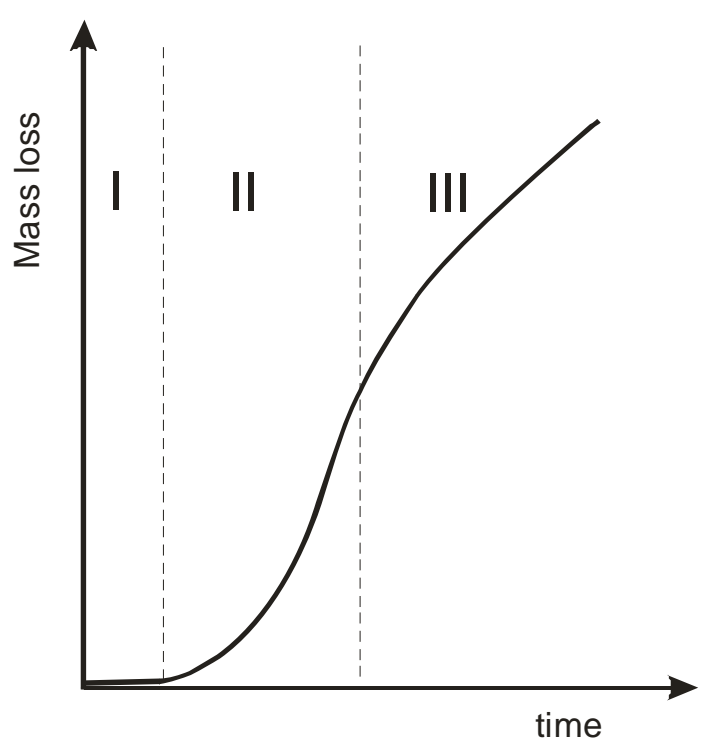

Fig. 1. Typical cavitation degradation curve

The cavitation erosion resistance of bulk materials increases with the increase of material hardness, fatigue resistance and also the reduction of grain size. For this reason the nanocrystalline materials, which possess high hardness, high fatigue resistance and very small grains, are supposed to have good cavitation resistance. Nanocrystalline Tibased coatings are the very common hard coatings used nowadays. Their impact wear [8-11], deformation behaviour [12] and cavitation resistance [13-16] have been investigated. Cavitation erosion investigations proved beneficial influence of nanocrystalline TiN coatings showing the importance of surface layer in initiating micro-cracks and their development. Although TiN coatings possess inner defects, which acted as nucleation centres for wear [13], most TiN coated specimens revealed improved cavitation erosion resistance compared to uncoated steel [13-16].

The elastic strain to failure, which is regarded as the ratio of hardness and elastic modulus and is identified as the key characteristic for wear resistance [8, 17], was also found as the main coating factor in cavitation resistance [16]. Leyland and Matthews [17] emphasized the significance of a low value of elastic modulus for the improvement of wear resistance of ceramic coatings. Carried out investigations of TiN and Ti-Al-N coatings Yoon et al. [9] concluded that the so-called "plasticity index" $\mathrm{H}^{3} /\left(\mathrm{E}^{*}\right)^{2}$, where $\mathrm{H}$ is hardness, $\mathrm{E}^{*}=\mathrm{E} /\left(1-\mathrm{v}^{2}\right) \mathrm{E}-$ Young's modulus, $\mathrm{v}-$ Poisson's coefficient, plays an 
important role in impact-wear behaviour of coating/substrate systems. They noticed that the impact-wear behaviour changed from plastic deformation to a brittle mode with the increase the "plasticity index". Similar results were obtained in Ref. [16]. Moreover, in Ref. [18] was proved the increase of cavitation erosion resistance of the $4 \mu \mathrm{m}$ thick TiN coatings deposited on the stainless steel with the decrease of the $\mathrm{H} \bullet \mathrm{L}_{\mathrm{C} 2}$ / E parameter, where $\mathrm{H}$ is coating hardness, $\mathrm{L}_{\mathrm{C} 2}$ - adhesion, $\mathrm{E}-$ Young's modulus of the TiN coating. Disadvantage of the most coatings deposited on conventional materials is the problem with good adhesion assurance. The cavitation erosion tests of chromium nitride coatings showed that coatings with weak adhesion have partly spalled-off [19]. Investigation performed by Krella and Czyżniewski [16] also confirmed that coatings with weak adhesion have partly spalled-off. Moreover, they achieved correlation adhesion with incubation period; an increase of critical load $\mathrm{L}_{\mathrm{C} 2}$ increased incubation period. The critical load $\mathrm{L}_{\mathrm{C} 2}$ is the load, at which the coating removal from inside of the scratch starts. The incubation period is believed to be the most important stage due to change in dislocation structure and initiation micro-cracks.

The aim of this paper is to analyse the degradation mechanism of the TiN coatings deposited at various parameters by means of the ARC PVD and with various thickness and propose the resistance parameter for the TiN coatings against cavitation action.

\section{EXPERIMENTAL TECHNIQUES}

The experimental tests were performed in a cavitation tunnel with a system of barricades. The schematic of the cavitation chamber is shown in Fig. 2. Cavitation intensity is controlled by varying the slot width and the boost pump speed. Flow conditions are defined by the $\mathrm{p}_{1}$ and $\mathrm{p}_{2}$ absolute pressures measured at the chamber inlet and outlet respectively. Slightly hard tap water $\left(5.9 \mathrm{mVal} / \mathrm{dm}^{3}\right)$ was used as the working liquid, temperature $20 \pm 2{ }^{\circ} \mathrm{C}$. The cavities (cavitating vortices and bubbles) are generated by the pressure decrease in the slot between two semi-cylindrical barricades. The substrates $(45 \mathrm{~mm} \times 26 \mathrm{~mm} \times 14 \mathrm{~mm})$ of the X6CrNiTi18-10 austenitic steel were subjected to quenching at $1050^{\circ} \mathrm{C}$. Substrates were ground and polished to the roughness of $\mathrm{R}_{\mathrm{a}} \leq 0.05 \mu \mathrm{m}$. The chemical composition of the X6CrNiTi18-10 steel is presented in Table 1 and basic properties in Table 2 . The nanocrystalline TiN coatings were deposited by cathodic arc evaporation method (ARC) [16]. The typical process of coating deposition consists of pumping down to a vacuum below $2 \times 10^{-3} \mathrm{~Pa}$, heating the substrates, cleaning the substrates by argon and titanium ions, initially deposition thin titanium interlayer $(\sim 0.1 \mu \mathrm{m}$ thickness $)$ in the argon atmosphere and finally deposition of TiN coating in a nitrogen atmosphere. Parameters applied to the deposition of TiN coatings are presented in Table 3 . The coated specimens were marked TiN-200, TiN350, TiN-500, where the number after the dash represents the substrate temperature during deposition and TiN-0, TiN-300, where the number after the dash represents bias voltage and TiN-8, TiN-12, where the number after the dash represents the coating thickness; uncoated specimen was marked with the symbol of the austenitic steel X6CrNiTi18-10. 


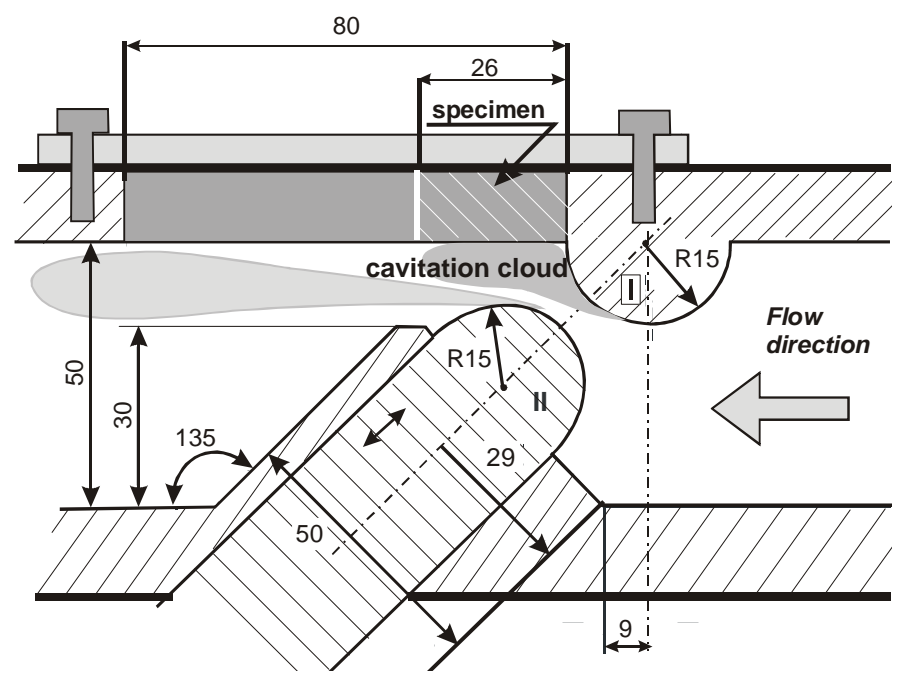

Fig. 2. Schematic view of cavitation chamber: I - stationary barricade, II - moving counter-barricade; the width of slot can be adjusted within the $0-15 \mathrm{~mm}$ range (all dimensions in $\mathrm{mm}$ )

Table 1. Chemical compositions of the X6CrNiTi18-10 steel, wt. pct

\begin{tabular}{|c|cccccccc|}
\hline & $\mathrm{C}$ & $\mathrm{Mn}$ & $\mathrm{Cr}$ & $\mathrm{Ni}$ & $\mathrm{Ti}$ & $\mathrm{Si}$ & $\mathrm{P}$ & $\mathrm{S}$ \\
\hline X6CrNiTi18-10 & 0.014 & 1.79 & 17.36 & 9.56 & 0.23 & 0.53 & 0.025 & 0.027 \\
\hline
\end{tabular}

Table 2. Properties of X6CrNiTi18-10 stainless steel

\begin{tabular}{|c|c|c|c|}
\hline $\begin{array}{c}\text { Hardness } \\
{[\mathrm{GPa}]}\end{array}$ & $\begin{array}{c}\text { Young's modulus } \\
{[\mathrm{GPa}]}\end{array}$ & $\begin{array}{c}\text { Ultimate tensile strength } \\
{[\mathrm{GPa}]}\end{array}$ & $\begin{array}{c}\text { Roughness }\left(\mathrm{R}_{\mathrm{a}}\right) \\
{[\mu \mathrm{m}]}\end{array}$ \\
\hline 1.7 & 199 & 0.684 & 0.02 \\
\hline
\end{tabular}

Table 3. Deposition parameters of TiN coatings [16]

\begin{tabular}{|l|l|}
\hline Pressure of residual gases & $2 \times 10^{-3} \mathrm{~Pa}$ \\
\hline Working pressure of argon & $1 \mathrm{~Pa}$ \\
\hline Working pressure of nitrogen & $1 \mathrm{~Pa}$ \\
\hline Arc current & $85 \mathrm{~A}$ \\
\hline Substrate bias voltage & $-300,-100, \sim 0 \mathrm{~V}$ \\
\hline Substrate temperature & $200,350,500^{\circ} \mathrm{C}$ \\
\hline
\end{tabular}

The phase compositions of coatings were tested on a DRON2 X-ray diffractometer using $\mathrm{CuK} \alpha$ radiation. The grain size was determined by means of the Scherrer method with reflex parameters (location and FWHM) using Gaussian analysis. The coating morphology was examined with JEOL JSM 5500 LV scanning electron microscope (SEM). The hardness and Young modulus were measured with a NanoHardness Tester (CSEM) using the method of Oliver and Pharr [20]. A scratch tester Revetest ${ }^{\circledR}$ 
produced by CSEM was used to investigate the coating adhesion. Basic properties of the TiN coatings deposited on X6CrNiTi18-10 steel are shown in Table 4. The H/E coefficient is the ratio of hardness $(\mathrm{H})$ and elastic modulus $(\mathrm{E})$. The critical load $\mathrm{L}_{\mathrm{C} 1}$ is defined by occurrence of the first cohesive failure of the coating, that is the cracks caused by tensile stresses inside and on the edges of the scratch behind the sliding diamond cone. The critical load $\mathrm{L}_{\mathrm{C} 2}$ is the load at which the coating removal from inside of the scratch starts

Table 4. Properties of TiN coatings

\begin{tabular}{|c|c|c|c|c|c|c|c|}
\hline & TiN-200 & TiN-350 & TiN-500 & TiN-0 & TiN-300 & TiN-8 & TiN-12 \\
\hline \multirow{2}{*}{$\begin{array}{l}\text { Deposition } \\
\text { parameters }\end{array}$} & -100 & -100 & -100 & $\sim 0$ & -300 & -100 & -100 \\
\hline & 200 & 350 & 500 & 350 & 350 & 350 & 350 \\
\hline $\begin{array}{l}\text { Hardness, H, } \\
\text { [GPa] }\end{array}$ & 26.8 & 21.7 & 20.8 & 22.9 & 25.1 & 26.1 & 27.4 \\
\hline $\begin{array}{l}\text { Young's modulus } \\
\text { E, [GPa] }\end{array}$ & 341.8 & 344.2 & 424.2 & 402.9 & 369.4 & 433 & 525 \\
\hline H/E coefficient & 0.078 & 0.063 & 0.049 & 0.057 & 0.068 & 0.0603 & 0.0522 \\
\hline $\begin{array}{l}\text { Coating thickness } \\
\text { h, }[\mu \mathrm{m}]\end{array}$ & 3.6 & 3.7 & 3.8 & 3.8 & 3.6 & 7.8 & 11.9 \\
\hline \multirow{2}{*}{ Adhesion $\frac{\mathrm{L}_{\mathrm{C}^{2}}[\mathrm{~N}]}{\left.\mathrm{L}^{2}\right]}$} & 10 & 10 & 10 & 10 & 10 & 12 & 10 \\
\hline & 15 & 23 & 14 & 30 & 24 & 33 & 34 \\
\hline
\end{tabular}

The results for the adhesion testing of TiN coatings are presented in Table 4. Taking into account the low hardness of the X6CrNiTi18-10 steel (about $2 \mathrm{GPa}$ ) and its large plastic deformation under substantial pressure of the Rockwell indenter, used for the scratch test, the examinations show good adhesion of the TiN coatings to this type of substrate. The first minor cracks occurred with a scratch made under the load of $10 \mathrm{~N}$ for most coatings and $12 \mathrm{~N}$ only for TiN-8 coating. It corresponds to the critical load $\mathrm{L}_{\mathrm{C} 1}$ defined by occurrence of the first cohesive failure of the coating, that is the cracks caused by tensile stresses inside and on the edges of the scratch behind the sliding diamond cone. The critical loads $\mathrm{L}_{\mathrm{C} 2}$ at which coating removal from inside of the scratch starts and coating's adhesion attests were in range from 14 to $34 \mathrm{~N}$ (Table 4).

The TiN coated specimens were subjected to cavitation impingement in the cavitation chamber operated with inlet pressure $\mathrm{p}_{1}=1000 \mathrm{kPa}$, outlet pressure $\mathrm{p}_{2}=130 \mathrm{kPa}$ and a slot width of $5 \mathrm{~mm}$. In order to obtain the erosion curves, the mass loss measurement was performed after each exposure interval. Before the test and after each test interval the specimens were cleaned, dried and reweighed. At the beginning of the cavitation test the measurements were conducted every $30 \mathrm{~min}$ of exposure (for the first $180 \mathrm{~min}$ of test) to estimate the incubation period. The duration of exposure intervals was then gradually increased. The total cavitation test duration was $600 \mathrm{~min}$. After cavitation test the cavitation erosion damage was analysed with the scanning electron microscope (SEM). 


\section{RESULTS}

The mass losses of the TiN coated X6CrNiTi18-10 stainless steel and of the uncoated X6CrNiTi18-10 steel during cavitation tests are shown in Fig. 3. The plotted data are the average of three specimens. The error is $\pm 10 \%$.

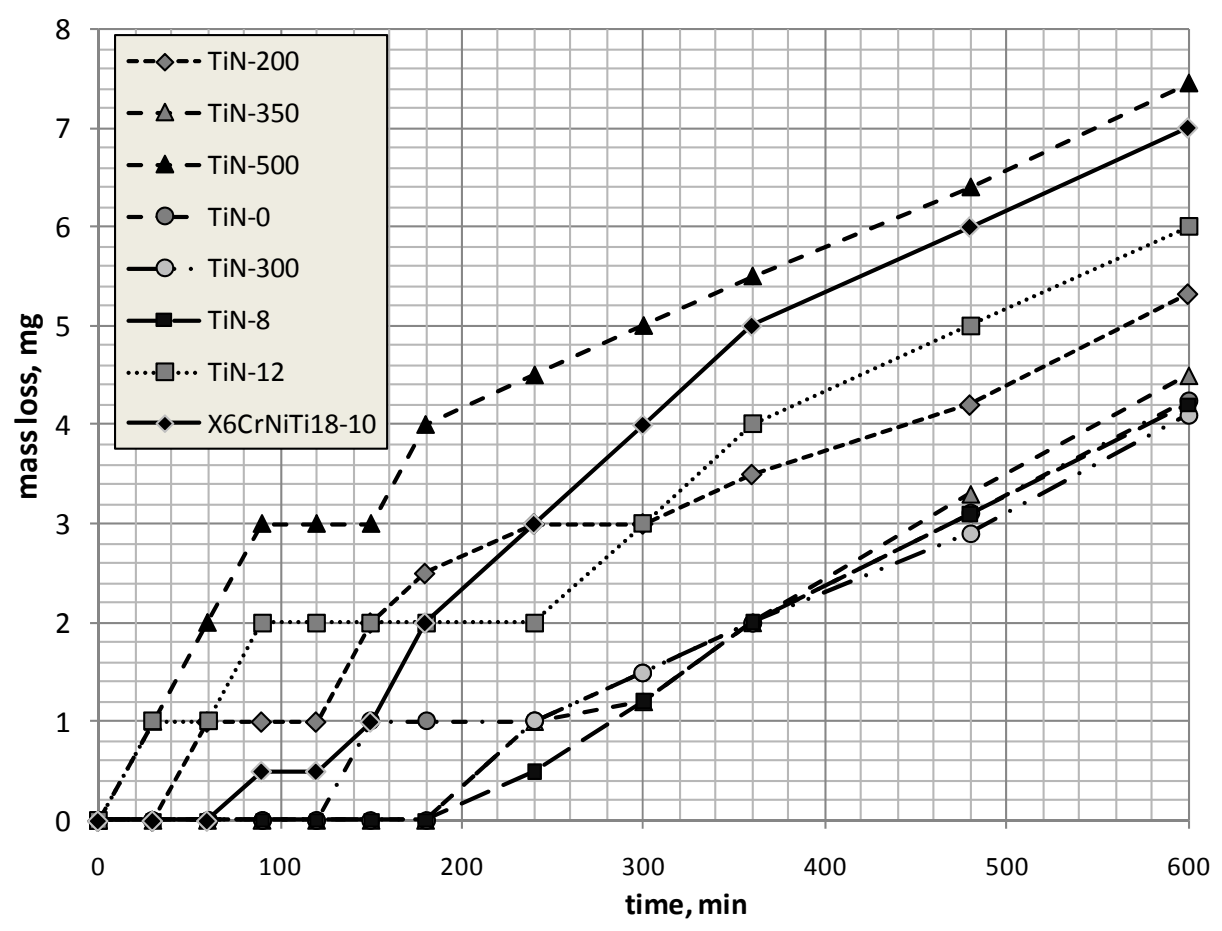

Fig. 3. Cavitation erosion curves

The investigations have shown that the parameters of TiN coating deposition had influence on coatings properties, incubation period and on mass loss. At incipient erosion the $\operatorname{Ti}(\mathrm{N})$ micro-droplets were removed from the coating surface. This was likely the reason of the mass loss of the TiN-500 coating, which had the most Ti(N) micro-droplets, at the beginning of the cavitation test. Moreover, on most specimens no mass loss took place for about 1 hour of exposure. The break in the mass loss of examined specimens might be connected with the phase transformation $\gamma \rightarrow \alpha$ ' of the austenitic stainless steel, which was used as the substrate. The energy delivered to the specimen was used for the phase transformation, not for initiation the cracks. As it was proved in the Ref. [15] the phase transformation $\gamma \rightarrow \alpha^{\prime}$ occurs despite the deposition of the TiN protecting coating. Such a break in the mass loss did not occur in case of the TiN-350, TiN-300 and TiN-8 specimens, whose incubation periods were much longer than of the other coatings. Probably during incubation period the phase transformation $\gamma \rightarrow \alpha$ ' occurred. Thus, the phenomena of the phase transformation elongated the incubation period of the TiN-350 and the TiN-300 coatings. Afterwards solid particles of hard coating or / and steel substrate - coating system were spalled. After 500 minutes of exposure cavitation curves of all coated specimens were parallel to uncoated stainless steel.

Nearly all the TiN coatings have undergone micro-undulation (Fig. 4), which was caused by collapsing bubbles in the vicinity of the solid surface, and was related to the 
substrate undulation. Implosion of collapsing cavitation bubble in the solid surface can cause plastic deformation in the form of a pit. Veovodin et al. [10] noticed that during the dynamic impact test the main reason for degradation of PVD hard coatings was substrate deformation, which took place under the impact, and which the hard and often brittle coating was not able to follow. In present investigations, the first cohesive fractures have occurred on the top of the micro-folding, and progressed mostly along the top and through concave traces of post-microdroplets.

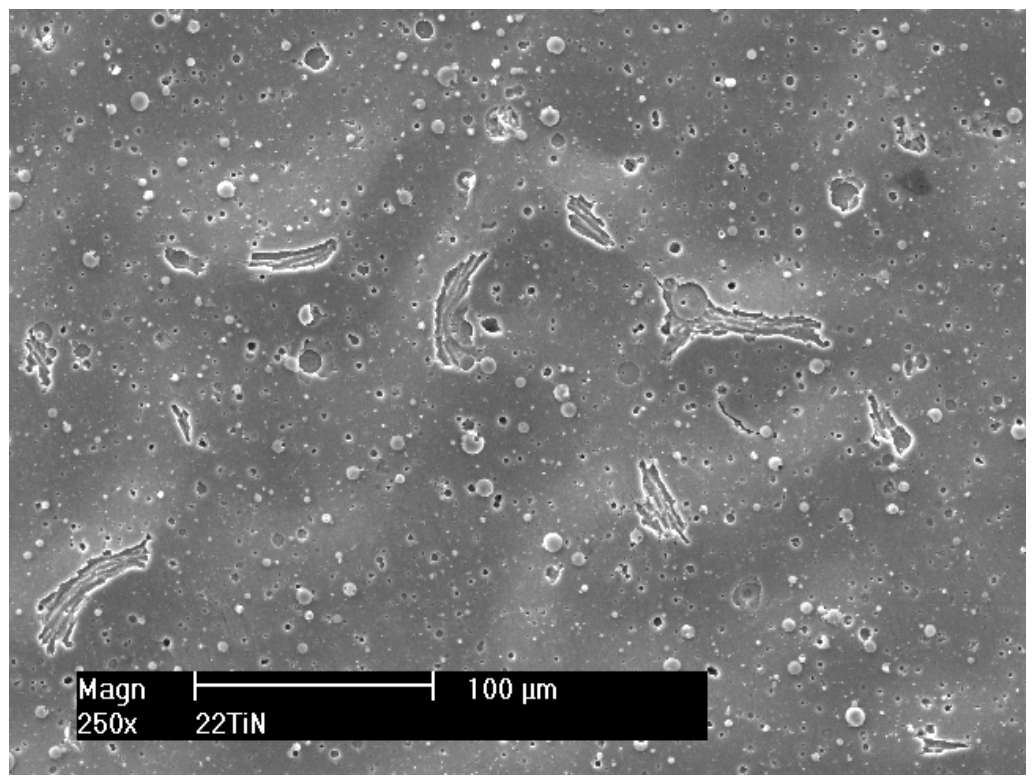

Fig. 4. A sample of the TiN coating undulation (SEM image of the TiN-350 coating)

Later, fractures have occurred at valleys (Fig. 5), probably due to delamination. Microscopic observations suggest that delamination occurred at the side of micro-folds. Moreover, Fig. 5 shows that the deformation process of the hard TiN coating goes via cracking (arrow). The PVD coatings possess the columnar structure, so the shown cracks were likely the results of grain boundary sliding. The grain boundary sliding allows the hard coating deforms and undergoes micro-undulation. Hard and brittle coating is not able to follow increasing deformation of the soft substrate and delaminate. Time to delamination depends on coating adhesion and plastic properties of the coating. If adhesion is low, e.g. in case of the TiN-500 coatings (Tab. 4), delamination will occur quickly. The TiN-500 coating was separated quite easily from the substrate (Fig. 6). At delaminated areas microcracks have occured early, especially when they were associated with some coating defects. The TiN-500 coating was spalled quite rapidly in a brittle manner. This was likely the reason of lack of the incubation period and high mass loss of the TiN-500 coatings. 


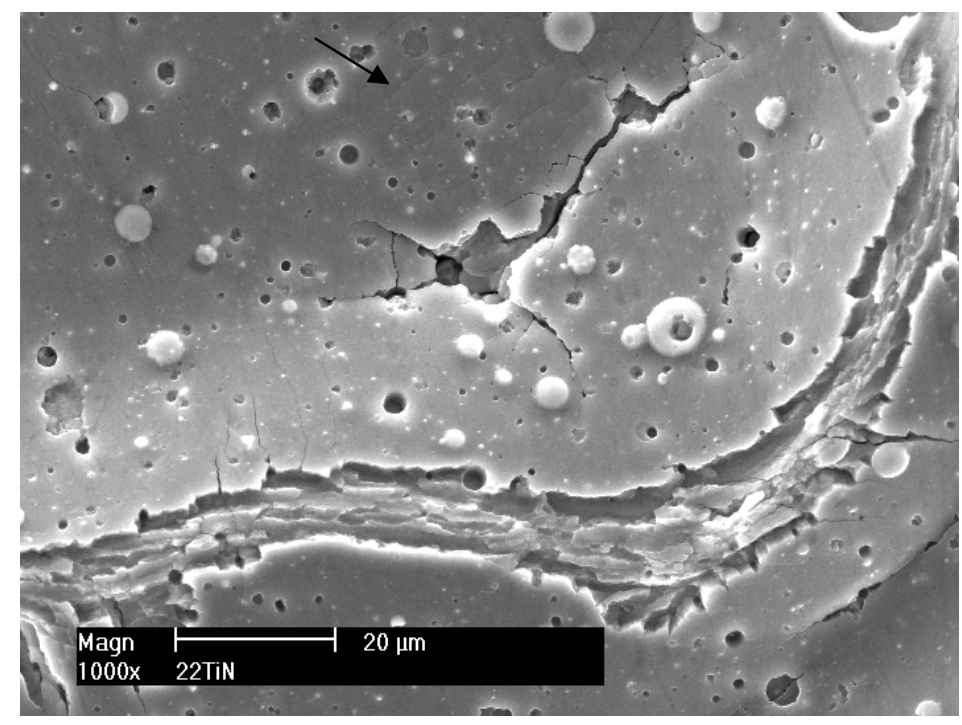

Fig. 5. Fracture at the valley (SEM image of the TiN-350 coating)

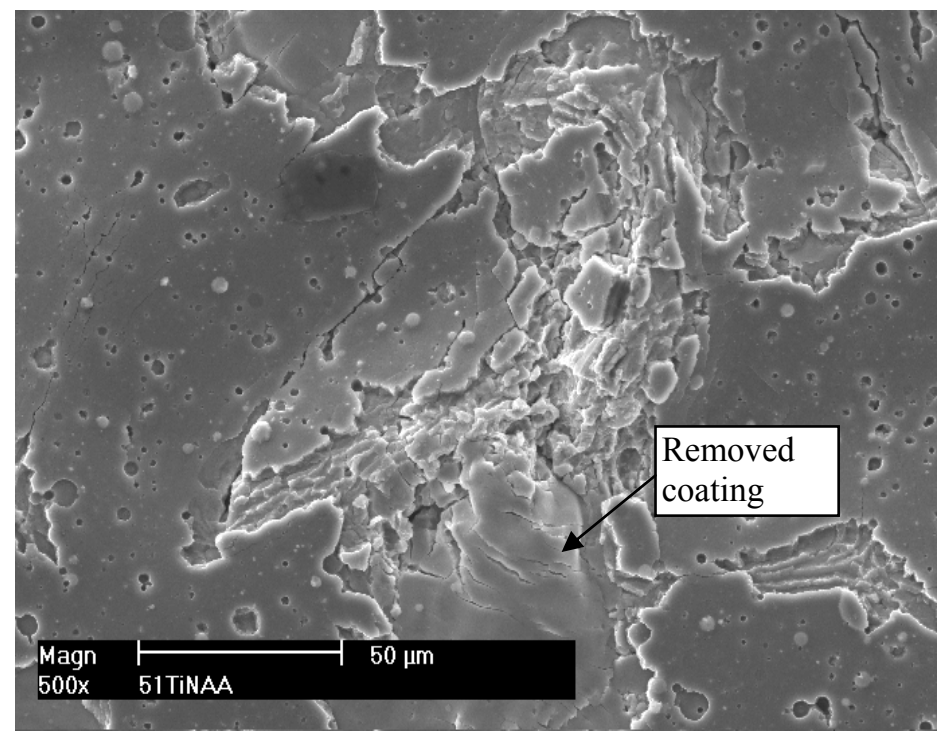

Fig. 6. SEM image of degradated TiN-500 coating after 600 min

Another places of microcracks initiation were the concave traces of the post- $\operatorname{Ti}(\mathrm{N})$ microdroplets and the boundary between the $\mathrm{Ti}(\mathrm{N})$ microdroplets and the TiN coating. Nevertheless, when the number of the concave traces of the post- Ti(N) micro-droplets was very high, e.g. in case of the TiN-0 coating (Fig. 7), cracks have developed from larger post- micro-droplets which were situated around the spots of the cavitation bubble implosion. Large number of small micro-droplets have not played major role in degradation process. Thus, the increase of the number of discontinuities spots did not affect the linear increase of the cracks initiation. This suggest that not all the discontinuity spots affect the crack initiation and adhesion and strength of the TiN coatings had higher influence on the cracks initiation and erosion rate then the coatings morphology. 


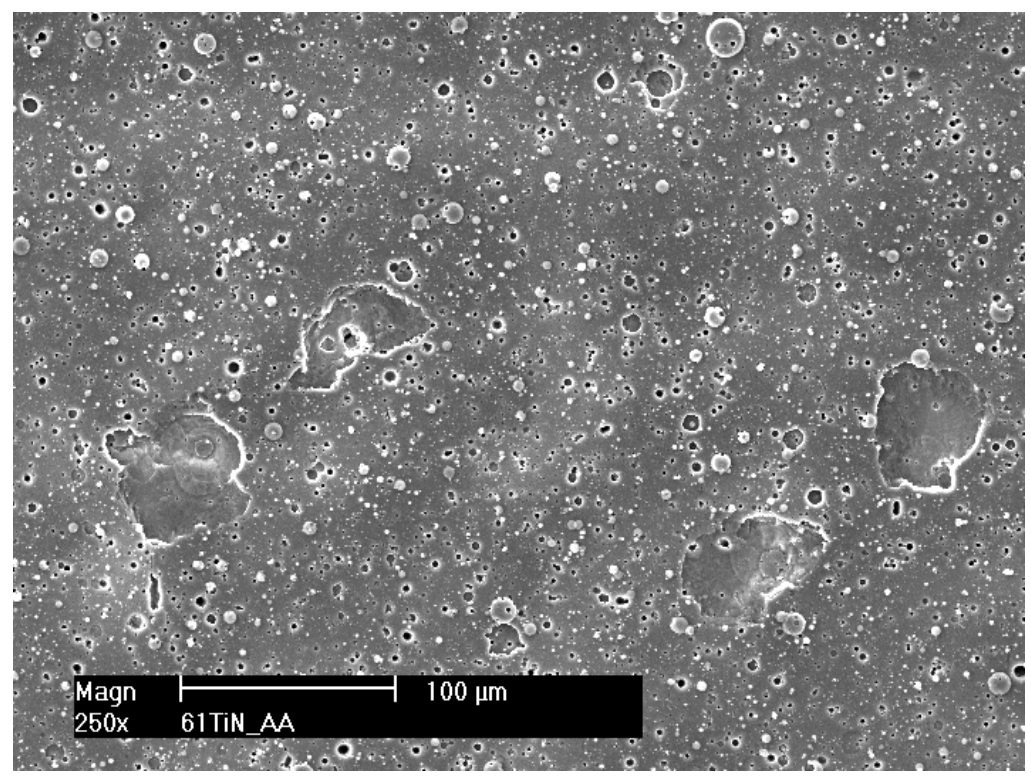

Fig. 7. SEM image of the surface of the TiN-O coating

The increase of the TiN coating thickness has caused the increase of the coating Young modulus, hardness and adhesion, although the plasticity index (H/E ratio) remain in the range of the rest tested coatings (Tab. 4). The degradation of the TiN-8 and the TiN-12 coatings differs from the TiN coatings of $4 \mu \mathrm{m}$ thick. The coating (Fig. 8) was removed in brittle mode in the form of thin flakes. The cracks have developed from the inner larger micro-droplets which were situated underneath the spot of the impact of cavitation pulse. In the Ref. [22] was proved that the place of crack initiation is correlated with the co-called "Bielajew's point", which is located under the surface of the impact and where the highest lateral stresses arose. Fig. 8 shows that cracks have typical feature of fatigue fracture for hard materials: cycling crack growth lines.

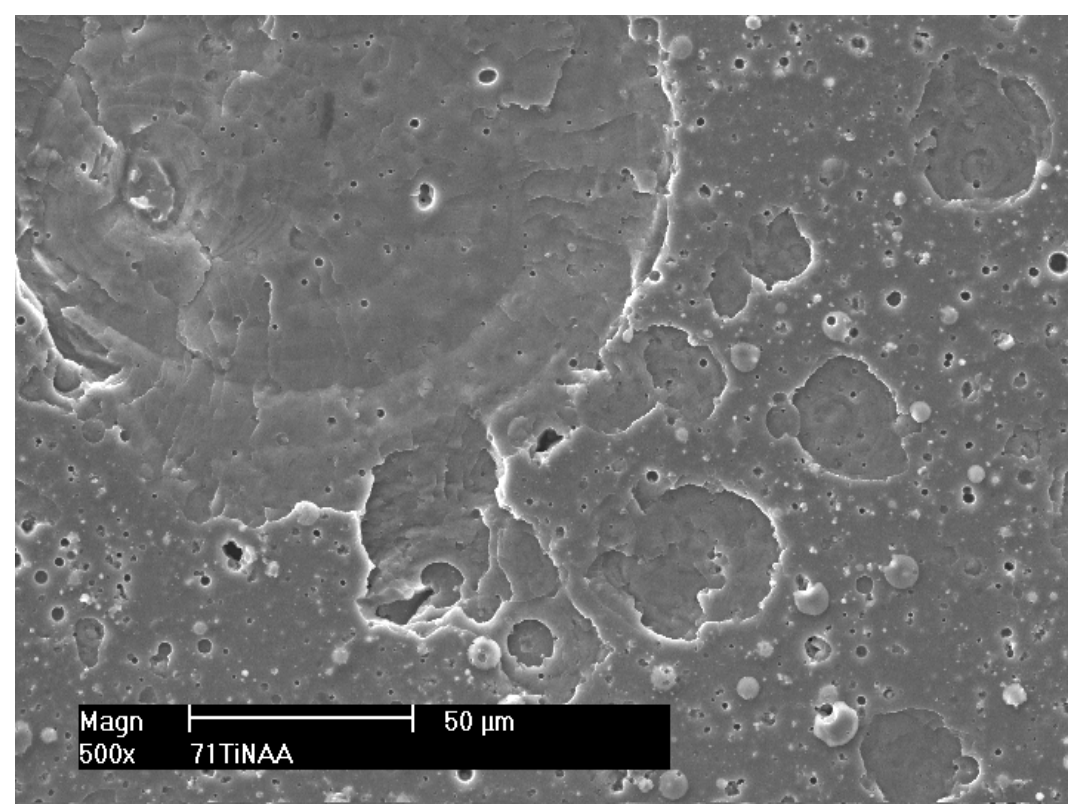

Fig. 8. SEM image of degradated TiN-8 coating after $600 \mathrm{~min}$ 


\section{DISCUSSION}

Under repeated implosions of cavitation bubbles, material degradation has a fatigue character. This means that every new impact can be considered as a new load cycle and rupture appears when material is solicited beyond its rupture limit. Deformation, which occurs at the early stage of degradation, is caused by dislocation motion. Dislocation investigation of austenitic stainless steel [7] showed that after 5 minutes of cavitation dense dislocation network developed, slip bands appeared in several slip systems and slip bands were crossed by cutting bands. Moreover, dislocation density and dislocation cells depend on energy supplied to the material by imploding bubbles. The investigation showed that significant energy is absorbed by material on plastic deformation. With test time, material absorbs more and more energy. After each cavitation pulse impact, new energy income leads to the increase of dislocation density. Simultaneously with surface undulation, material hardness increases. When dislocation density is so high that they cannot move anymore, every new energy income from each solicitation cycle causes material fracture.

Erosion of the TiN coatings occurred via rupture and spalling of coating particles. Places of the TiN coating rupture were: top of the undulation, delamination spots and discontinuity spots. Most cracks originated at discontinuity spots showing the destruction role of the micro-droplets. In case of the 4 microns TiN coatings from the micro-droplets located at the top of the undulation the cohesive cracks developed (Fig. 4). Ma et al [12] have found that plastic deformation of TiN coating occurred via cracking and sliding along the inter-columnar grain boundaries at contact surface with the substrate, where the highest stresses are. This proves that the highest tensile stresses were at the top of micro-folds, especially at discontinuity spots, and those stresses exceeded strength of the TiN coating. Cracks propagated through discontinuity spots leading to adhesive cracks and to spalling of coating fragments.

In case of the TiN coatings with thickness of 7.8 and $11.9 \mu \mathrm{m}$ along with the increase of coating thickness the number of inner discontinuity spots increases as well. They were the inner spots of stress pile-up and in some way played the role of inner cracks, according to the theory of fracture. Most discontinuity spots were the micro-droplets. If their dimension is above the critical size then the cracks can developed. The theory of fracture says that the larger the inner crack the less stress is needed to expand the crack and cause the rapid fracture. Moreover, the critical stress needed to the uncontrolled crack propagation is reciprocal proportional to the square root of the crack radius. Thus, the increase of the coating thickness causes non-linear increase of the spots of the micro-cracks development and the coating resistance to fracture is reciprocal related to the square root of the crack thickness.

The investigations show that as long as the TiN coatings can adjust to deformed substrate without cohesive or adhesive cracks, they protect the substrate and lessen the mass loss. According to Ref. [8, 9, 17], the H/E ratio is used to assess the plasticity of hard coatings. With the increase of the $\mathrm{H} / \mathrm{E}$ ratio the deformation mode changes from ductile to brittle. When a coating is very ductile (very low $\mathrm{H} / \mathrm{E}$ ratio), every little energy income causes dislocation movement and residual deformation. Time to block dislocation is short. Moreover, elastic limit and rupture limit are usually also low. Thus, repeated impacts of cavitation pulses lead to low-cycle fatigue, which implies that macro plastic deformation occurs in every loading cycle. Time to reach the rupture stress at a given point is short, so the coating cannot sustain the new energy income. 
Thus it breaks and lead to the accelerated erosion. Therefore, cavitation erosion resistance of very ductile coatings is low.

As the hardness and Young modulus increase, then more energy is needed to move dislocation. Thus, a coating absorbs more energy for work-hardening and the adjustment to a substrate. Time to reach the rupture stress lengthens. Thus, cavitation resistance of a given material increases. In case of very hard (brittle) and stiff materials energy needed to cause dislocation motion and plastic deformation is higher than energy needed to initiate brittle fracture. For that reason microcracks in very hard materials occur faster than plastic deformation. Thus, erosion of extremely hard and stiff materials accelerates rapidly.

Next place of the cracks initiation were delamination spots. This shows that adhesion plays an important role in the protection of the substrate against cavitation: delaying the erosion process. Performed investigation confirmed that adhesion had influence on both the incubation period and the mass loss. Batista et al. [21] attributed the improvement in cavitation and impact resistance to very good adhesion of the tested coating. Carney et al. [11] and Ma et al [12] obtained that under cyclic loading the substrate deformation occurs faster than the TiN coating deformation especially if there is large difference in hardness between the substrate and the TiN coating. This leads to the TiN coating delamination.

Cavitation pulses can be described as high-velocity nano- or mikro-indentiations. Under the impact, the coating and the substrate are bent, so in the substrate high tensile and shear stresses arose. After unloading the coating and the substrate relax. Because of the difference in hardness and in the elastic modulus of the coating and the substrate, the rate of relaxation of the coating differs from the substrate's one. In case of the TiN coating and the X6CrNiTi18-10 substrate, the TiN coating tends to relax faster than the substrate. The adhesion counteracts it and causes that the coating and the substrate relax together. At the end of the relaxation stage the coating is stressed elastically, while the substrate can be deformed plastically. Some shear stresses remain along the coating substrate interface; the highest at the sides of a pit of the earlier deformed substrate. Every load cycle increases the deformation of the TiN coating due to an adjustment to the more deformed substrate. Moreover, every load cycle increases the residual stresses. When at a given point the residual stress exceeds the shear strength, shear failure occurs. The hard TiN coating deforms via shearing along the columnar grain boundaries. The shear strength at the inter-columnar boundary depends on hardness and Young's modulus of the coating, while the shear strength at the interface depends on the adhesion. The improvement of the TiN coating adhesion allows the coating to accommodate to some extent the substrate deformation. Thus, every new cycle leads to (i) the increase of cohesive fracture in the coating and (ii) the increase of the delamination.

Taking into consideration all aforementioned key factors the $\mathrm{H} \cdot \mathrm{L}_{\mathrm{C} 2} / \mathrm{E} \cdot \mathrm{h}^{1 / 2}$ parameter was obtained. Correlation between the $\mathrm{H} \cdot \mathrm{L}_{\mathrm{C} 2} / \mathrm{E} \cdot \mathrm{h}^{1 / 2}$ parameter and the mass loss is shown at Fig. 9. The increase of the $\mathrm{H} \cdot \mathrm{L}_{\mathrm{C} 2} / \mathrm{E} \bullet h^{1 / 2}$ parameter decreases the mass loss of steel substrate - hard coating system. The obtained curve has shows a regular descent indicating a continuous improvement in the erosion resistance with the increase of the $\mathrm{H} \cdot \mathrm{L}_{\mathrm{C} 2} / \mathrm{E} \cdot \mathrm{h}^{1 / 2}$ parameter. The trend line correlates perfectly with all experimental points $\left(\mathrm{R}^{2}=0.9638\right)$. Additionally, similar relationship was also obtained for the mass loss after 360 and $300 \mathrm{~min}$ of exposure and the $\mathrm{H} \cdot \mathrm{L}_{\mathrm{C} 2} / \mathrm{E} \bullet \mathrm{h}^{1 / 2}$ parameter. 


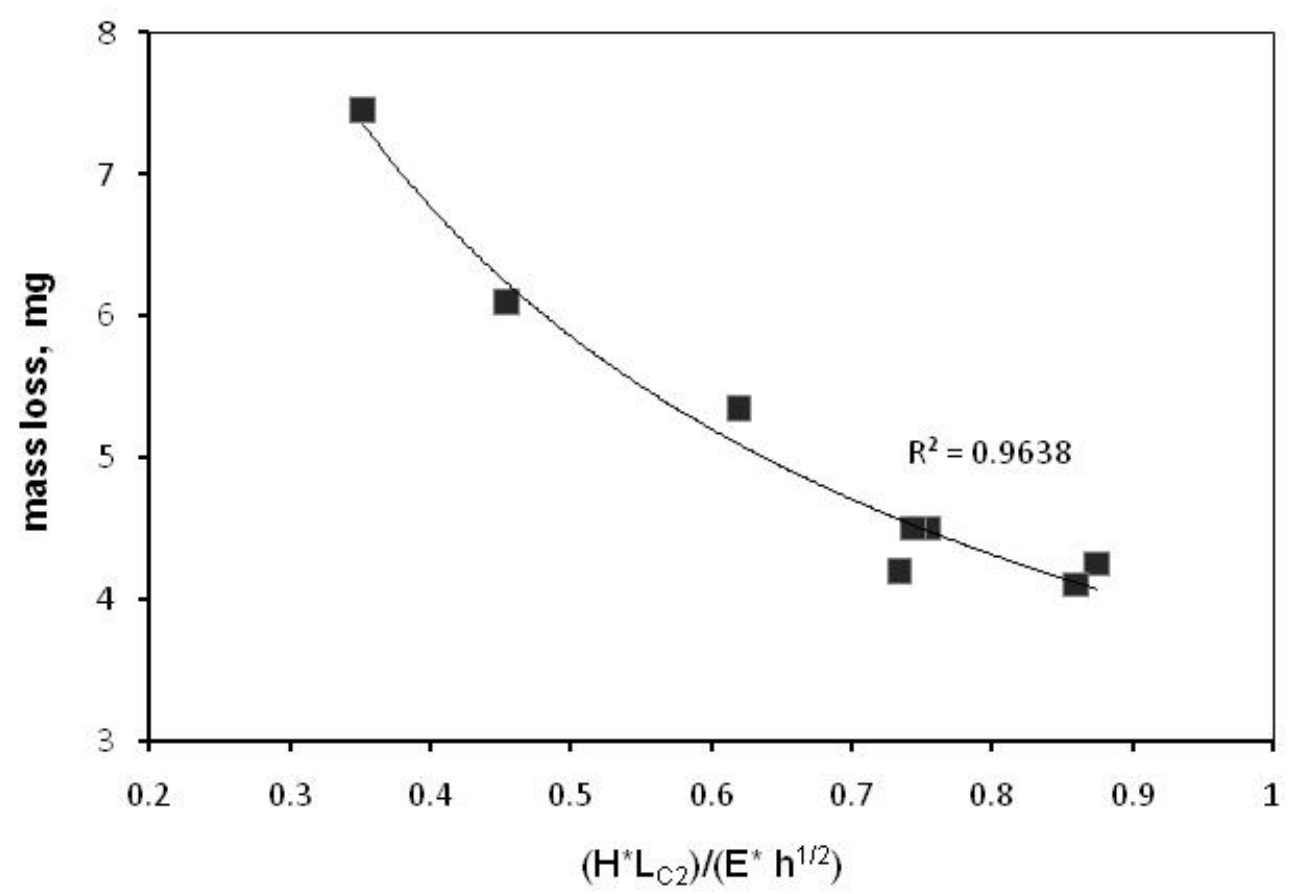

Fig. 9. Correlation between the $\mathrm{H} \bullet \mathrm{L}_{\mathrm{C} 2} / \mathrm{E} \cdot \mathrm{h}^{1 / 2}$ parameter and the mass loss of the TiN coatings

\section{CONCLUSIONS}

The study presents results of cavitation tests of the TiN hard coatings deposited on the X6CrNiTi18-10 austenitic steel by ARC PVD method. It was affirmed that the mass loss of the TiN coatings is related to adhesion, plastic deformation of the coatings and inner coatings defects, which are correlated with the coatings thickness.

The obtained results and their analysis allow the following conclusion:

- The TiN coatings undergo micro-folding at the early stage of cavitation erosion. Microscopic observations revealed that the first micro-cracks appeared at top of micro-folding and at delamination spots.

- The new $\mathrm{H} \cdot \mathrm{L}_{\mathrm{C} 2} / \mathrm{E} \cdot \mathrm{h}^{1 / 2}$ parameter, which joints the essential factor in cavitation resistance of steel substrate - hard coating system, shows very good fitting to date points. The curve had a regular descent indicating a continuous improvement in the erosion resistance with the increase of the $\mathrm{H} \bullet \mathrm{L}_{\mathrm{C} 2} / \mathrm{E} \bullet \mathrm{h}^{1 / 2}$ parameter.

\section{ACKNOWLEDGEMENTS}

The author wishes to thank warmly dr Andrzej Czyżniewski, Koszalin University of Technology, Institute of Mechatronics, Nanotechnology and Vacuum Technique, for the deposition of TiN coatings, and for performing the adhesion measurements. 


\section{REFERENCES}

1. Brennen Ch.E., Cavitation and bubble dynamics, The Oxford Engineering Science Series 44, Oxford University Press (1995).

2. Knapp R.T., Resent Investigations of cavitation and cavitation damage, Trans. ASME 77 (1955) 1045-1054.

3. Philipp A., Lauterborn W., Cavitation erosion by single laser-produced bubbles, J.Fluid Mech. 361 (1998) 75-116.

4. Bourne N.K., On the collapse of cavities, Shock Waves 11 (2002) 447-455.

5. Tabor D.: A theory of static and dynamic hardness. Engineering 165 (1948) 289292.

6. Bourne N.K., Millett J.C.F., Gray III G.T., On the shock compression of polycrystalline metals, J. Mater. Sci. 44 (2009) 3319-3343.

7. Krella A., Influence of cavitation intensity on X6CrNiTi18-10 stainless steel performance in the incubation period. Wear 258 (2005) 1723-1731.

8. Iwai Y., Honda T., Yamaa H., Matsumura T., Larsson M., Hogmark S.: Evaluation of wear resistance of thin hard coatings by a new solid particle impact test. Wear (2001),pp. 861-867.

9. Yoon S.Y., Yoon S.Y., Chung W.S., Kim K.H.: Impact-wear behaviors of TiN and Ti-Al-N coatings na AISI D2 steel and WC-Co substrates. Surface and Coating Technology 177-178 (2004), pp.645-650.

10. Voevodin A.A., Bantle R., Matthews A.: Dynamic impact wear of $\mathrm{TiC}_{\mathrm{x}} \mathrm{N}_{\mathrm{y}}$ and TiDLC composite coatings. Wear 185 (1995), pp.151-157.

11. Cairney J.M., Tsukano R., Hoffman M.J., Yang M.: Degradation of TiN coating under cyclic loading. Acta Materialia 52 (2004), pp. 3229-3237.

12. Ma L.W., Cairney J.M., Hoffman M., Munroe P.R.: Deformation mechanisms operating nanoindentation of TiN coatings on steel substrates. Surface \& Coatings Technology 192 (2005), pp.11-18.

13. Münsterer S., Kohlhof K.: Cavitation protection by low temperature TiCN coatings. Surface and Coatings Technology 74-75 (1995), pp. 642-647

14. Marynin V.H.: Erosion of vacuum-arc Ti-N coatings. Materials Science 39 (2003), pp. $447-451$

15. Krella A., Czyżniewski A.: Influence of the substrate hardness on cavitation erosion resistance of TiN coating. Wear 263 (2007), pp. 395-401.

16. Krella A., Czyżniewski A.: Investigation concerning the cavitation resistance of TiN coatings deposited on austenitic stainless steel at various temperatures. Wear 265 (2008), pp. 72-80.

17. Leyland A., Matthews A.: On the significance of the H/E ratio in wear control: a nanocomposite coating approach to optimized tribological behaviour. Wear 246 (2000), pp. 1-11. 
18. Krella A., The influence of TiN coatings properties on cavitation erosion resistance, Surface \& Coatings Technology 204 (2009) pp. 263-270.

19. Han S., Lin J.H., Kuo J.J., He J.L., Shih H.C.: The cavitation-erosion phenomenon of chromium nitride coatings deposited using cathodic arc plasma deposition on steel. Surface and Coating Technology 161 (2002), pp. 20-25.

20. Oliver W.C., Pharr G.M.: An improved technique for determining hardness and elastic modulus using load and displacement sensing indentation experiments. J Mater Res 7 (1992), pp. 1564-1583.

21. Batista C.A., Godoy C., Matthews A., Impact testing of duplex and non-duplex (TiN, Al)N and Cr-N PVD coatings, Surface and Coatings Technology 163-164 (2003), pp. 353-361.

22. Krella A.: Cavitation resistance of TiN nanocrystalline coatings with various thickness, Advances in Materials Science. Vol. 9, No. 2 (20), June 2009, pp.12-24. 